\title{
Both endurance training and strength training reduced disability and pain in chronic non-specific neck pain in women
}

Ylinen J, Takala EP, Nykänen M, et al. Active neck muscle training in the treatment of chronic neck pain in women: a randomized controlled trial.JAMA 2003;289:2509-16.

QUESTION: In women with chronic non-specific neck pain, does intensive isometric neck strength training or lighter endurance training of neck muscles reduce pain and disability?

\section{Design}

Randomised \{allocation concealed*\}†, blinded \{data collectors, outcome assessors, data analysts, data safety and monitoring committee, and manuscript writers $\} \nmid{ }^{*}$ controlled trial with 1 year of follow up.

\section{Setting}

Occupational healthcare services in Finland.

\section{Patients}

180 female office workers 25-53 years of age (mean age 46 y) who were permanent employees, had constant or frequently occurring neck pain for $>6$ months, and were motivated to continue working and receive rehabilitation. Exclusion criteria included severe disorders of the cervical spine, frequent migraine, peripheral nerve entrapment, fibromyalgia, shoulder diseases, and inflammatory rheumatic diseases. Follow up was $98 \%$ at 1 year.

\section{Intervention}

Women were allocated to 1 of 3 groups: strength training $(n=60)$, endurance training $(n=60)$, and control $(\mathrm{n}=60)$. Training regimens consisted of 5 weekly 45 minute sessions for 12 days, with instructions for continuing the exercises at home. The strength group used an elastic band to do high intensity, isometric neck strengthening and stabilisation exercises. The endurance group exercised the neck flexor muscles by lifting the head from supine and prone positions. Both groups used dumbbells to do dynamic exercises for the shoulders and arms and ended the session with 20 minutes of stretching. The control group spent 3 days every 2 months at the rehabilitation centre doing recreational activities. All 3 groups were instructed to do 30 minutes of aerobic exercise 3 times a week.

Source of funding:

Social Insurance

Institution, Helsinki,

Finland.

For correspondence:

DrJ Ylinen, Jyväskylä

Central Hospital,

Jyuäskylä, Finland.

jari.ylinen@ksshp.fi

Abstract and

commentary also

appear in ACP Journal

Club.

Strength or endurance training $v$ no training in women with chronic neck pain at 1 year:

\begin{tabular}{lllll} 
Outcome & Comparisons & Event rates & RBI (95\% CI) & NNT (CI) \\
Complete pain relief & Strength $v$ control & $73 \% v 20 \%$ & $261 \%(120$ to 521$)$ & 2 (2 to 3$)$ \\
\hline & Endurance $v$ control & $59 \% v 20 \%$ & $188 \%(71$ to 405$)$ & $3(2$ to 5$)$ \\
\hline
\end{tabular}

\section{Main outcome measures}

Neck pain and disability (visual analogue [VAS] scale, modified neck and shoulder pain and disability index, Vernon neck disability index, and 6 -point scale $[1=$ much more pain, $6=$ complete relief from pain]).

\section{Main results}

Analysis was by intention to treat. Each training group had greater improvement on the VAS scale, neck and shoulder pain and disability index, and the Vernon disability index than did the control group. More patients in each training group than in the control group had complete pain relief (table).

\section{Conclusion}

In women with chronic non-specific neck pain, both intensive isometric neck strength training and lighter endurance training of neck muscles reduced disability and pain.

*See glossary.

$\dagger$ Information provided by author.

\section{COMIMENTARY}

The study by Ylinen et al had a sound design, was well executed, and had near perfect follow up. The 2 exercise groups improved more than the control group on most outcome measures. It is important to note that both exercise groups also had a multimodal rehabilitation programme, which included training in relaxation and ergonomics, behavioural support to reduce fear of pain and increase motivation to exercise, and 4 sessions of physical therapy (massage and mobilisation) for pain relief. The control group had none of these. Hence, a more accurate title for this review and the article itself would be "Multimodal rehabilitation programme reduced disability and pain in chronic non-specific neck pain."

The authors comment that these programmes, without ongoing home exercises, have shown only short term responses in the previous studies. Hence, they attribute the durable responses in this study to the home exercises. This may be the case, but a study design where home exercise is the only difference between groups is required for this conclusion.

An interesting difference, which may be attributed to the type of exercise, appears between the 2 exercise groups: the strength group had greater increases in neck strength and rotation range than did the endurance group, but relief of pain and strength were similar.

When applying the results to clinical practice, readers should note closely the many exclusion criteria and the enviable rates of compliance with the strength and endurance exercises throughout the follow up year. According to participants' diaries, this averaged 2 of the recommended 3 times per week. Such good compliance may have been motivated by trial staff or the exercise diaries themselves or may have been inflated by the participants keen to please the investigators on paper. None the less, this high compliance marks this study as one of efficacy in optimal conditions rather than effectiveness in usual practice. Even if such good compliance with exercise could be achieved in clinical practice, gaining access to such an intensive and efficacious programme may be challenging in many countries.

Michael Yelland, MBBS, FRACGP

University of Queensland, Herston, Queensland, Australia 